\title{
Geometric phase in coupled neutron interference loops
}

\author{
Y. Hasegawa, M. Zawisky, and H. Rauch \\ Atominstitut der Österreichischen Universitäten, Schüttelstraße 115, A-1020 Wien, Austria \\ A. I. Ioffe* \\ Berlin Neutron Scattering Center, Hahn-Meitner Institut, Glienickerstraße 100, D-14109 Berlin, Germany
}

(Received 10 October 1995)

\begin{abstract}
A geometric phase factor is derived for a split-beam experiment as an example of cyclic evolutions. Poincare sphere descriptions for the split-beam experiment show its geometric property. We observe this geometric phase with a two-loop neutron interferometer, where a reference beam can be added to the beam from one interference loop. The combination of phase shifters and partial absorbers permitted the compensation of the dynamical phase and measurement of the geometric phase. All the experimental results show complete agreement with our theoretical treatment. We discuss a situation where a geometric phase exists even when the dynamical phase becomes zero.
\end{abstract}

PACS number(s): 03.75.Dg, 03.65.Bz, 07.60.Ly, 61.12.Ld

\section{INTRODUCTION}

During the past decade, the geometric effect on the phase of the wave function has excited considerable interest. It was Berry [1] who first clearly described the geometric phase factor for a quantum system transported adiabatically through a curve $C$ in parameter space, this phase factor depending solely upon the geometry of the curve $C$. Simon [2] reformulated this phase in terms of a line bundle over the parameter space. The geometric phase is closely related to the phase discovered by Pancharatnam [3] in the 1950s. Several experiments were reported to manifest the effect of this geometric phase. A spinning light experiment in an optical fiber [4] was the first of this kind. A similar experiment was accomplished with a neutron beam in an adiabatically rotating magnetic field $[5,6]$. Other techniques yielded further observations, e.g., nuclear quadrupole resonance [7] and electron diffraction [8]. Aharanov and Anandan [9] released the restriction of adiabaticity for Berry's phase so that the geometric phase may be generalized to the state of the system in a cyclic evolution, i.e., that it returns to its initial state after an evolution. Experiments to observe this AharanovAnandan (AA) geometric phase were accomplished by laser interferometry $[10,11]$ and nuclear magnetic resonance [12]. In addition, a dynamical aspect of the evolving geometric phase [13] and the geometric effect for noncyclic evolution [14] were demonstrated experimentally. More recently, other examples were shown of geometric phases in cyclic population transfer between two atomic states [15] and in cyclic excursion around a diabolic point [16].

It is well-known that the spinor rotation of a spin- $\frac{1}{2}$ particle in a homogeneous magnetic field results in a geometric phase $[1,9]$. Two bases, spin-up and spin-down states, are assumed in such a case. In a split-beam experiment [17-19] where an incident beam is split and recombined, one can insert phase shifters and/or absorbers into each split beam, so

\footnotetext{
*Permanent address: St. Petersburg Nuclear Physics Institute, Gatchina, 188350, Russia.
}

that the system evolves under the action of two separate Hamiltonians. In these circumstances, two similar bases are observable. This similarity justifies regarding neutron interferometry, which is an example of the split-beam experiment, as a cyclic evolution of the quantum system. Thus, we can define the dynamical and geometric phase factors for the system inside the interferometer. In this case, the phase shifter, which is inserted to observe the interference oscillations, directs the evolution of the system along a certain curve $C$ and the absorber, which reduces the intensity of one beam, changes this curve of the evolution. Since it is necessary to add a reference beam to the beam, which is recombined from one interference loop, we utilized a four-plate neutron interferometer with two loops [20] to observe this geometric phase.

In this paper, we justify the split-beam experiment as a cyclic evolution of a quantum system by analogy to the spinor rotation. In our theoretical treatment, the overall phase is considered as a sum of weighted phases of the two superposed partial beams, and the dynamical and the geometrical phase factors are derived for the cyclic evolution. Its geometrical property is shown with the use of Poincaré sphere descriptions. The four-plate neutron interferometer with two interference loops enabled to realize experiments to observe this geometric phase and the experimental results completely agree with the theoretical treatment.

\section{PRINCIPLE OF THE EXPERIMENT}

\section{A. Cyclic evolution of the split-beam experiment}

The spinor rotation of a spin- $\frac{1}{2}$ particle in a constant magnetic field provides a good example of the cyclic evolution of a quantum system in which the system returns to the original state after a certain evolution [9]. This spinor rotation can be described by assuming two bases, namely "up" and "down" spin eigenstates, and by assigning appropriate phase shifts due to the magnetic field. The system returns to the original state with a specific total phase shift $\phi$ when the difference between those two phase shifts is equal to an integral mul- 


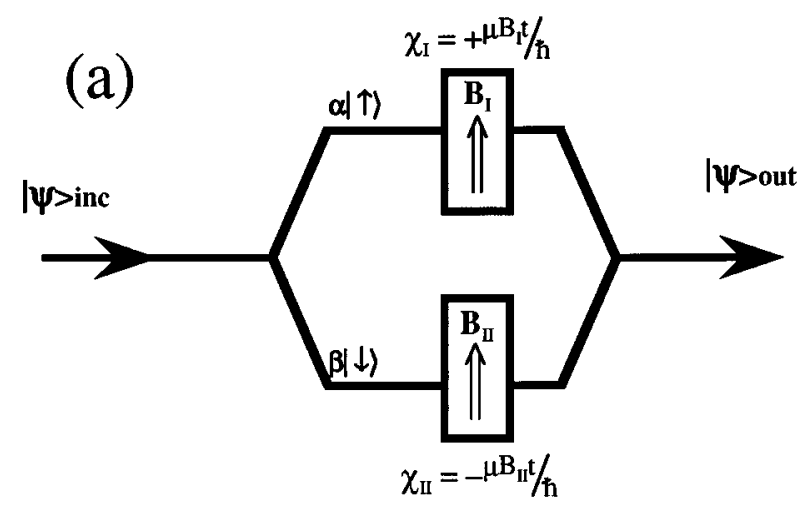

(b)

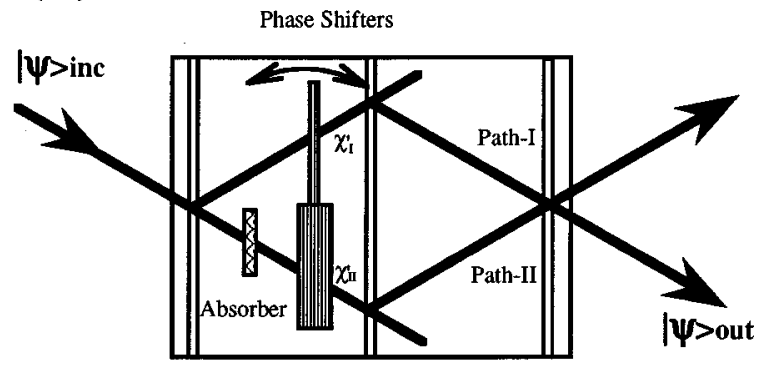

FIG. 1. Similarities between the spinor rotation of a spin- $\frac{1}{2}$ particle and a split-beam experiment. (a) A model of the spinor rotation. An incident beam is split into two spin states, affected by magnetic fields, $\mathbf{B}_{i}$, and recombined. Each beam undergoes the phase shift, $\chi_{i}$. (b) Schematic of a split-beam experiment for neutrons. An incident beam is split into two beam paths and recombined. A phase shifter, which induces phase shifts, $\chi_{i}^{\prime}$ is inserted into each beam and an absorber into one.

tiple of $2 \pi$. In this description, $2 \pi$ periodicity of the phase factor assures the cyclic evolution of the system.

It is instructive to conceptualize this spinor rotation within the framework of a coherent splitting of the incident beam [21] [Fig. 1(a)]. In this case, the incident beam is split into two beams of spin eigenstates, after which the split beams go through homogeneous magnetic fields and are recombined. The homogeneous magnetic fields $\mathbf{B}_{i}$ induce phase shifts $\chi_{\mathrm{I}}$ and $\chi_{\mathrm{II}}$ on the respective beams, which are given by $\chi_{\mathrm{I}}=+\mu B_{\mathrm{I}} t / \hbar$ and $\chi_{\mathrm{II}}=-\mu B_{\mathrm{II}} t / \hbar$ [9]. The spin state of the recombined beam depends on the difference between these phase shifts, $\chi_{\mathrm{I}}-\chi_{\mathrm{II}}$, and this spinor rotation is periodic with a period of $\chi_{\mathrm{I}}-\chi_{\mathrm{II}}=2 \pi$.

Similar circumstances are present in the split-beam experiment for neutrons shown in Fig. 1(b). We assume a conventional triple-Laue interferometer for neutrons [17-19], where an incident beam is split at the first plate of the interferometer, and the two resulting beams are reflected at the second plate and recombined at the third. Several optical components can be inserted in each split beam path, such as a phase shifter or a beam attenuator. We can change the amount of the phase shifts, $\chi_{\mathrm{I}}^{\prime}$ and $\chi_{\mathrm{II}}^{\prime}$, e.g., by rotating the phase shifter. The intensities of the two outgoing beams are modulated complementarily, dependent on the phase differ- ence, $\chi_{\mathrm{I}}^{\prime}-\chi_{\mathrm{II}}^{\prime}$, between the two split beams in the interferometer. It should be noted that the intensities of these two split beams oscillate periodically with a period of $\chi_{\mathrm{I}}^{\prime}-\chi_{\mathrm{II}}^{\prime}$ $=2 \pi$. In the split-beam experiment, there are two base vectors, one for each of the two split beams. If these two base vectors are attributed to two spin eigenstates in the spinor rotation, we can define dynamical and geometric phases in the split-beam experiment by analogy to established terminology.

\section{B. Geometric phase in the split-beam experiment}

The geometric phase has already been defined in the cyclic evolution of the spinor rotation by removing the dynamical part from the total phase shift [9]. Suppose that a Hamiltonian $H^{\prime}$ in the rest frame propagates a normalized state $|\Psi(t)\rangle$ along a closed curve. The dynamical phase $\Phi_{D}$ is then given by

$$
\Phi_{D}=-\hbar^{-1} \int_{0}^{\tau}\left\langle\Psi(t)\left|H^{\prime}\right| \Psi(t)\right\rangle d t
$$

With this dynamical phase, the geometric phase $\beta$ is given by

$$
\beta=\phi-\Phi_{D} .
$$

where $\phi$ represents the total phase shift of the system during the cyclic evolution. It is worth noting here that Eq. (1) can be rewritten in the form

$$
\Phi_{D}=\int_{0}^{\tau}\langle\Psi(t)|-\Delta \omega d t| \Psi(t)\rangle=\int_{0}^{\tau}-\Delta \omega d t
$$

for the normalized state $|\Psi(t)\rangle$, where $\Delta \omega$ represents the shift of the angular frequency due to $H^{\prime}$.

The similarities between the spinor rotation and the splitbeam experiment allow us to define the dynamical phase for the split-beam experiment in an analogous way. It is a conventional procedure to calculate the phase shift for the propagation of the neutron beam through a material in terms of a change of the wave vector due to its index of refraction. Moreover, the phase of the wave function is given by $(\mathbf{k} \cdot \boldsymbol{l}$ $-\omega t)$. Thus, we derive here the dynamical phase in the splitbeam experiment in terms of the change of the wave vector, $\Delta \mathbf{k}$. Considering that the normalization of the incident beam is sometimes destroyed by the beam attenuation due to an absorber, as shown in the setup in Fig. 1(b), the dynamical phase $\Phi_{D}^{\prime}$ can be defined as

$$
\begin{aligned}
\Phi_{D}^{\prime}= & \frac{\int_{l}\langle\Psi|\Delta \mathbf{k} \cdot d \boldsymbol{l}| \Psi\rangle}{\langle\Psi \mid \Psi\rangle}=\frac{\int_{l_{\mathrm{I}}}\left\langle\Psi_{\mathrm{I}} \mid \Psi_{\mathrm{I}}\right\rangle \Delta \mathbf{k}_{\mathrm{I}} \cdot d \boldsymbol{l}}{\langle\Psi \mid \Psi\rangle} \\
& +\frac{\int_{l_{\mathrm{II}}}\left\langle\Psi_{\mathrm{II}} \mid \Psi_{\mathrm{II}}\right\rangle \Delta \mathbf{k}_{\mathrm{II}} \cdot d \boldsymbol{l}}{\langle\Psi \mid \Psi\rangle},
\end{aligned}
$$

where $\left|\Psi_{i}\right\rangle, \boldsymbol{l}_{i}$, and $\mathbf{k}_{i}$ represent the two wave functions, beam paths, and wave vectors in the interferometer, respectively. Here, we omit the real part of the phase shift due to the absorber, which does not reduce the general validity of our treatment. Rearranging Eq. (4), we get 


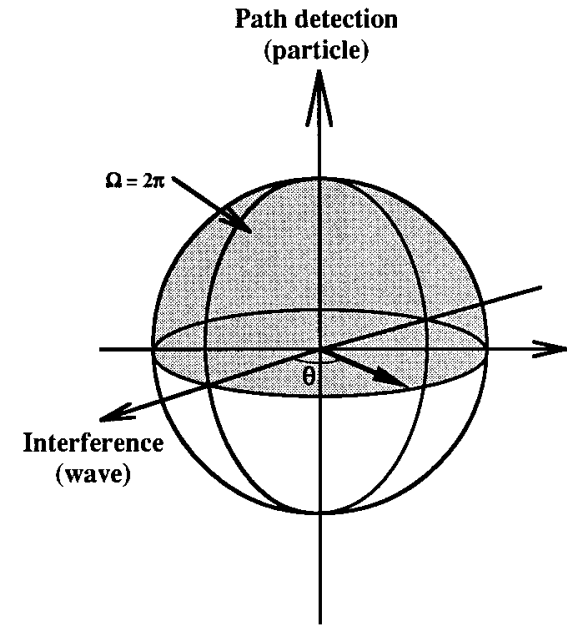

(a)

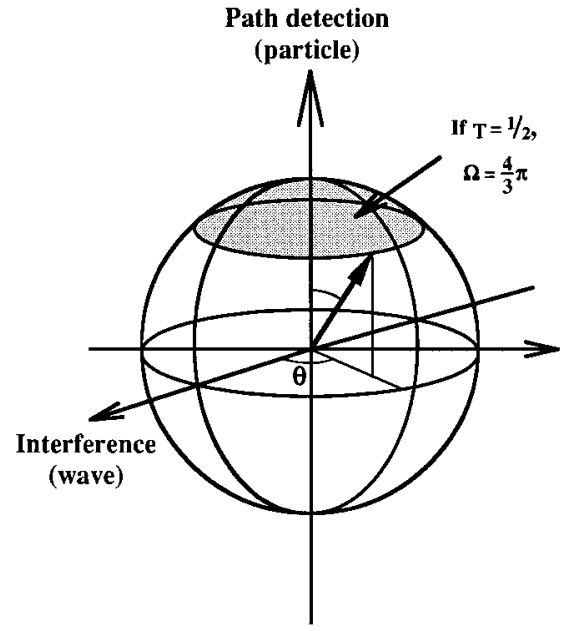

(b)

$$
\Phi_{D}^{\prime}=\frac{I_{\mathrm{I}}}{\left(I_{\mathrm{I}}+I_{\mathrm{II}}\right)} \chi_{\mathrm{I}}^{\prime}+\frac{I_{\mathrm{II}}}{\left(I_{\mathrm{I}}+I_{\mathrm{II}}\right)} \chi_{\mathrm{II}}^{\prime}=\left(\frac{1}{1+T}\right)\left(\chi_{\mathrm{I}}^{\prime}+T \chi_{\mathrm{II}}^{\prime}\right),
$$

where $I_{i}$ and $\chi_{i}^{\prime}$ represent intensities and phase shifts of the two beams, respectively, and $T$ is the transmission probability of the absorber in the beam path II.

The geometric phase $\beta^{\prime}$ in the split-beam experiment is derived in the same manner as Eq. (2) and is given with the total phase shift $\phi^{\prime}$ as

$$
\beta^{\prime}=\phi^{\prime}-\Phi_{D}^{\prime}
$$

It is useful to point out that we can choose the cyclic evolution in the split-beam experiment, so that the second term of Eq. (6) becomes zero. This may be regarded as an alternative definition of $\beta^{\prime}$. From Eq. (5), one can see that, when rotating the phase shifter, the dynamical phase shift $\Delta \Phi_{D}^{\prime}$ during the cyclic evolution becomes zero when

$$
\Delta \chi_{\mathrm{I}}^{\prime}+T \Delta \chi_{\mathrm{II}}^{\prime}=0
$$

where $\Delta \chi_{i}^{\prime}$ is the change of $\chi_{i}^{\prime}$ during the cyclic evolution. This equation shows that $\beta^{\prime}$ is explicitly observable in the split-beam experiment with the right combination of phase shifters and absorbers.

\section{Poincaré sphere description for the split-beam experiment}

It is very instructive to use the Poincare sphere for the split-beam experiment and thereby to recognize the geometric nature of the derived geometric phase just as the spinsphere was used for the spinor evolution. The split-beam experiment can be completely described within the framework of a two-dimensional Hilbert space $\mathrm{H}_{2}$, where the states can be visualized as elements of the Poincare sphere [22-25]. These spheres are shown in Fig. 2(a) for the cases of the equal beam intensities and 2(b) for the unequal beam intensities due to the absorber. The vertical axis represents the relative intensity of the two beams, and the polar points represent the single-beam situations. When shifting the rela-
FIG. 2. Poincaré sphere descriptions for splitbeam experiments for (a) the equal beam intensity condition, i.e., without an absorber. The state traces on the equator of the sphere, which yields the solid angle of $2 \pi$. (b) depicts the condition with unequal beam intensities, i.e., with an absorber. The state traces on a latitudinal circle on the sphere, which gives a certain solid angle according to the transmission probability, $T$, of the absorber. For instance, the solid angle is given by $\frac{4}{3} \pi$, when $T=\frac{1}{2}$.

tive phase $\theta$ between the two beams, the state in (a) traces on the equator of the sphere and the state in (b) traces a latitudinal circle on the sphere dependent on the ratio between the intensities of the two beams.

With these spheres, the solid angle $\Omega$, which is subtended by the traced curve at the origin, is given by

$$
\Omega=2 \pi\left(1-\frac{1-T}{1+T}\right)=4 \pi \frac{T}{1+T} .
$$

The geometric phase $\beta^{\prime}(C)$ for one cycle curve $C$ is associated with its solid angle $\Omega(C)$. Berry [1] has shown that this is given with the helicity $\sigma$ by

$$
\beta^{\prime}(C)=\sigma \Omega(C)=4 \pi \sigma \frac{T}{1+T} .
$$

Equation (8) shows that the solid angle $\Omega$ depends only on the transmission probability $T$ of the absorber, which determines the curve of the evolution, and the geometric phase $\beta^{\prime}(C)$ is derived from this transmission probability.

\section{Geometric phase measurement with a two-loop neutron interferometer}

We have derived the geometric phase in the split-beam experiment in the previous sections. When one uses the right combination of phase shifters and absorbers so that the condition of Eq. (7) is satisfied, the geometric phase emerges explicitly in the outgoing beam from one interference loop. The conventional one-loop triple-Laue neutron interferometer is suitable to elicit the geometric phase in the split-beam experiment. This type of interferometer, however, is of no use to observe and measure this geometric phase, since only the absolute square of the wave function of the relevant beam can be measured with it, and this hides the geometric phase shift.

This geometric phase can only be detected by an additional reference beam in an interference experiment. A fourplate neutron interferometer with two loops is the most suitable tool for this purpose [20]; the experimental setup is 


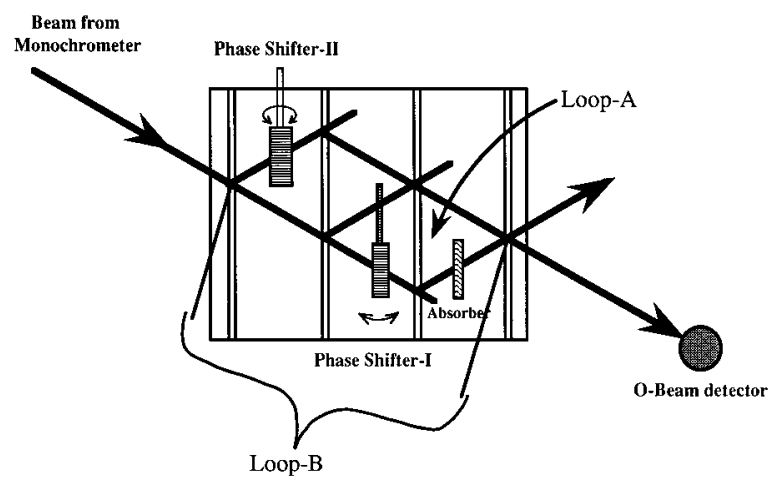

FIG. 3. Experimental setup to measure the geometric phase in the split-beam experiment with a four-plate neutron interferometer. The phase shifter I along with the absorber is inserted into an interference loop (loop $A$ ). In this loop, while phase shifters influence the evolution of the state, an absorber can change the curve of this evolution. The phase shifter II is inserted into the reference beam in the other interference loop (loop $B$ ) to observe the geometric phase as a shift of the interference oscillations.

shown in Fig. 3. In the interference loop (loop $A$ ) between the second and the fourth plate of the interferometer, appropriate pairs of phase shifters and absorbers are inserted in each split beam path to compensate for the dynamical phase during the cyclic evolution. The phase shifters direct the state of the recombined beam from this interference loop through cyclic evolutions and the absorber changes the curves of these cyclic evolutions. In the other interference loop (loop $B)$, a beam split at the first plate of the interferometer is recombined with the beam from the interference loop $A$. This split beam acts as a reference and another phase shifter is inserted in this beam path. The interference oscillations between the reference and the interference beam are measured using this additional phase shifter. The geometric phase of the outgoing beam from the interference loop $A$ is measured as shifts of these interference oscillations.

\section{EXPERIMENT}

\section{A. Apparatus}

The experiments were performed with the neutron interferometer instruments V9 at the BENSC, Hahn-Meitner Institut in Berlin [26]. A schematic view of the whole experimental arrangement is shown in Fig. 3. A four-plate neutron interferometer of monolithic perfect silicon crystal having two interference loops [20] was used. One interference loop (loop A) between the second and the fourth plates of the interferometer, is used to cause the evolution of the geometric phase. The other loop (loop $B$ ), which has an additional phase shifter, is used to observe the shifts of oscillations due to the geometric phase. This interferometer was adjusted to give (220) reflections. The 220 planes were perpendicular to the plates' surfaces. The wavelength was $1.95 \AA$, which gave a Bragg angle of $30.5^{\circ}$. The beam cross section was reduced to $2 \mathrm{~mm}$ (horizontal) and $10 \mathrm{~mm}$ (vertical) by a Cd diaphragm in front of the interferometer. As is shown in Fig. 3, a ${ }^{3} \mathrm{He}$ detector was set at one of the beams, this being in the transmitted direction after having been recombined from three beams, which have passed through various phase shifters, since this configuration was expected to show best
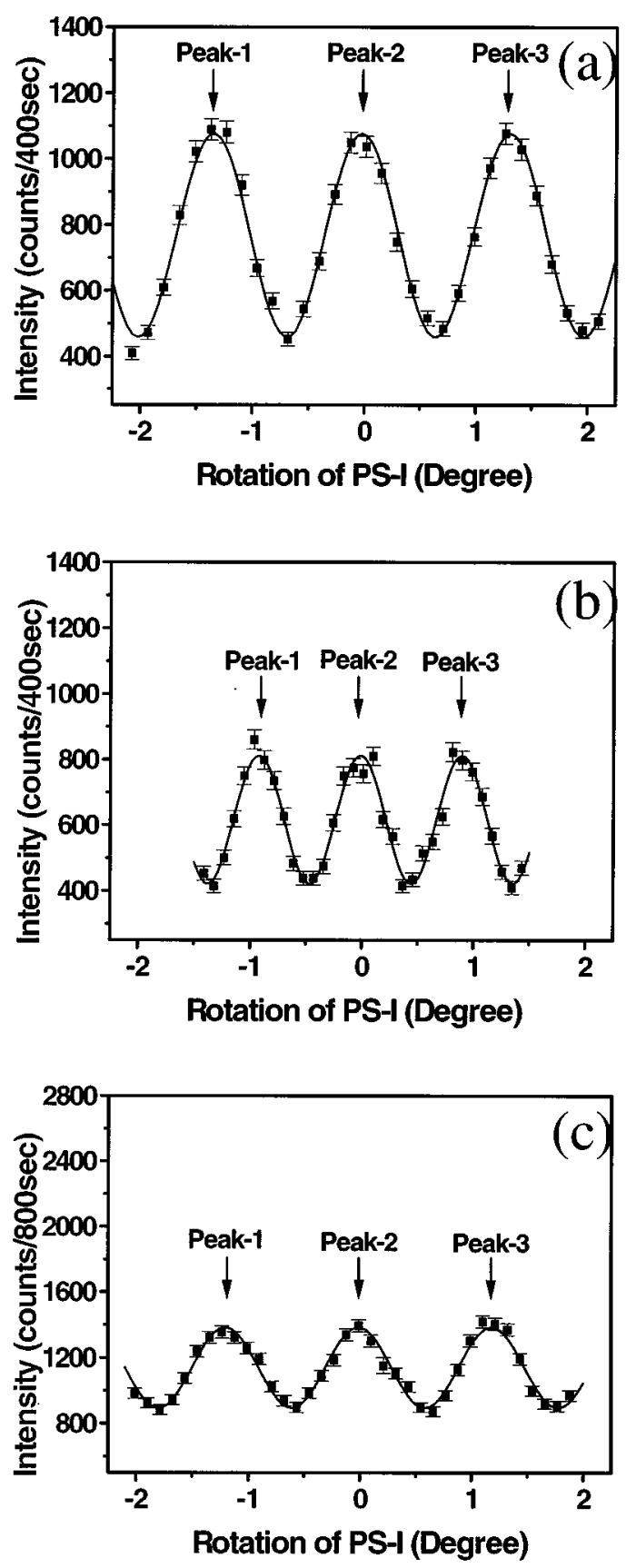

FIG. 4. Results of oscillation measurements performed by rotating the phase shifter I for the condition (a) without the absorber, (b) with the absorber having a transmission probability of 0.49 , and (c) with the absorber having a transmission probability of 0.21 .

contrasts for the interference loops concerned. We call this the $O$ beam detector. In this detector, the typical counting rate of the background was about $0.627(8)$ counts/sec.

In the interference loop $A$, an absorber and a phase shifter-which had different thicknesses for the two beams-were inserted. The absorber reduced the intensity of one of the beams, thereby changing the geometry of the evolution of the state. The relative phase between the two beams was changed by the phase shifter to promote the evolution of the state on a latitudinal circle on the sphere. Both situations are clearly described with Poincaré spheres in Fig. 2. 

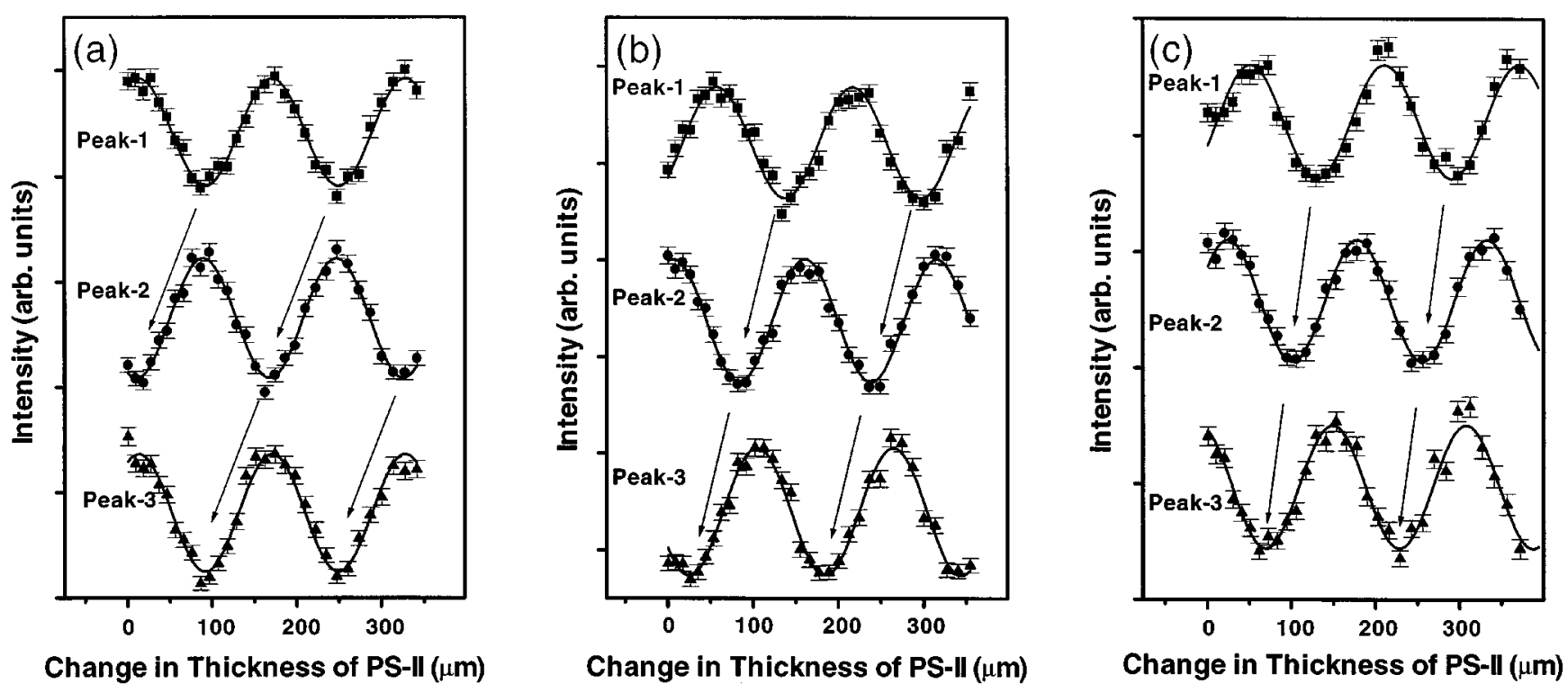

FIG. 5. Typical shifts of interference oscillations measured with the phase shifter II at the three peak positions and same conditions syndicated in Figs. 4(a)-(c). The extent of the shifts is reduced according to the decrease in the transmission probability of the absorber.

At first, no absorber was inserted in order to examine the case when the intensities of two beams were the same. Later we inserted two different kinds of absorbers in one of the beam paths between the third and the fourth plates of the interferometer. One was a gold-foil absorber $1 \mathrm{~mm}$ in thickness with the transmission probability $T=0.492(14)$. The other was a 1-mm-thick gold foil combined with a 1-mmthick indium foil. The transmission probability of $0.212(15)$ was achieved with this coupled absorber.

A phase shifter, called the phase shifter I (PS-I), was inserted between the second and third plates of the interferometer. Three kinds of parallel-sided $\mathrm{Al}$ plates were used, these having different thicknesses according to the transmission probability of the absorbers, so that the dynamical phase shifts during the evolution were zero. The first of these plates was $5 \mathrm{~mm}$ in thickness for both split beams and was used for the case without the absorber. The second was $10-\mathrm{mm}$ thick for the beam which had been reduced in intensity by the absorber and 5-mm thick for the other beam, and was used for the case in which the transmission probability of the absorber was 0.49 . The third was 10 -mm thick for the intensityreduced beam and 2-mm thick for the other, and was used for the case in which the absorber had a transmission probability of 0.21 . Rotation of these Al plates around the vertical axis produced a phase shift $\Delta \chi_{i}=-N \lambda b_{c} \Delta D_{i}$ on each beam, where $N$ is the number of nuclei per volume, $\lambda$ is the wavelength of neutrons, $b_{c}$ is the coherent scattering length of $\mathrm{Al}$ and $\Delta D_{i}$ is the change in thickness when the $\mathrm{Al}$ plate is rotated. Under these circumstances, the dynamical phase shift for the first case was assumed to be zero, and those for the second and the third cases were calculated to be roughly 0.06 and $0.15 \mathrm{rad}$, respectively, for one cycle of the evolution, i.e., for one period of the interference oscillations.

The beam in the interference loop $B$, which was split at the first plate of the interferometer and recombined with the beams from the other interference loop $(\operatorname{loop} A)$ at the fourth plate, is used as a reference for the phase. In order to obtain an adjustable phase reference, a parallel-sided $\mathrm{Al}$ plate which was $5-\mathrm{mm}$ thick was inserted in the reference beam path between the first and the second plates of the interferometer. We call this plate the phase shifter II (PS-II). Rotation of this $\mathrm{Al}$ plate around the horizontal axis changed the effective thickness of this plate in the beam and thus introduced the phase shift.

\section{B. Interference oscillations using the phase shifter I}

Before measuring the shifts of the interference oscillations with the phase shifter II, it was necessary to show with the phase shifter I how the interference loop $A$ would behave with various pairs of phase shifters and absorbers. In these measurements, an additional intensity modulations due to the interference loop $B$ should be avoided. Thus, the reference beam with the phase shifter II was blocked by inserting a Cd plate in this beam path between the first and the second plates of the interferometer. Interference oscillations with three different pairs of phase shifters I and absorbers were measured by rotating the phase shifter I.

Typical oscillations with least-squares fits for the three cases are shown in Fig. 4. In these three interferograms, the lower limit of the vertical axis indicates the background counting rate within the corresponding collecting time and the zero points of the horizontal axis are set to the central peaks of the oscillations, i.e., shifts of the oscillations due to the absorber and/or due to differences of the three different PS-I are omitted here. We denote the three peaks of each oscillation as peak 1, peak 2, and peak 3 . The contrast of the sinusoidal oscillation in the case without absorber, shown in Fig. 4(a), was about $60 \%$ after subtracting the background rate. One can easily see the reduction of the amplitude of oscillations in accordance with the decrease in the transmission probability of the absorber, which is due to the amplitude reduction of the beam by the absorber $[27,28]$. Since the three phase shifters I had different thicknesses, the periods of the oscillations were varied accordingly. For the following experiments, it was very important to determine their periods in order to cause the evolution of the system by exactly one cycle. 

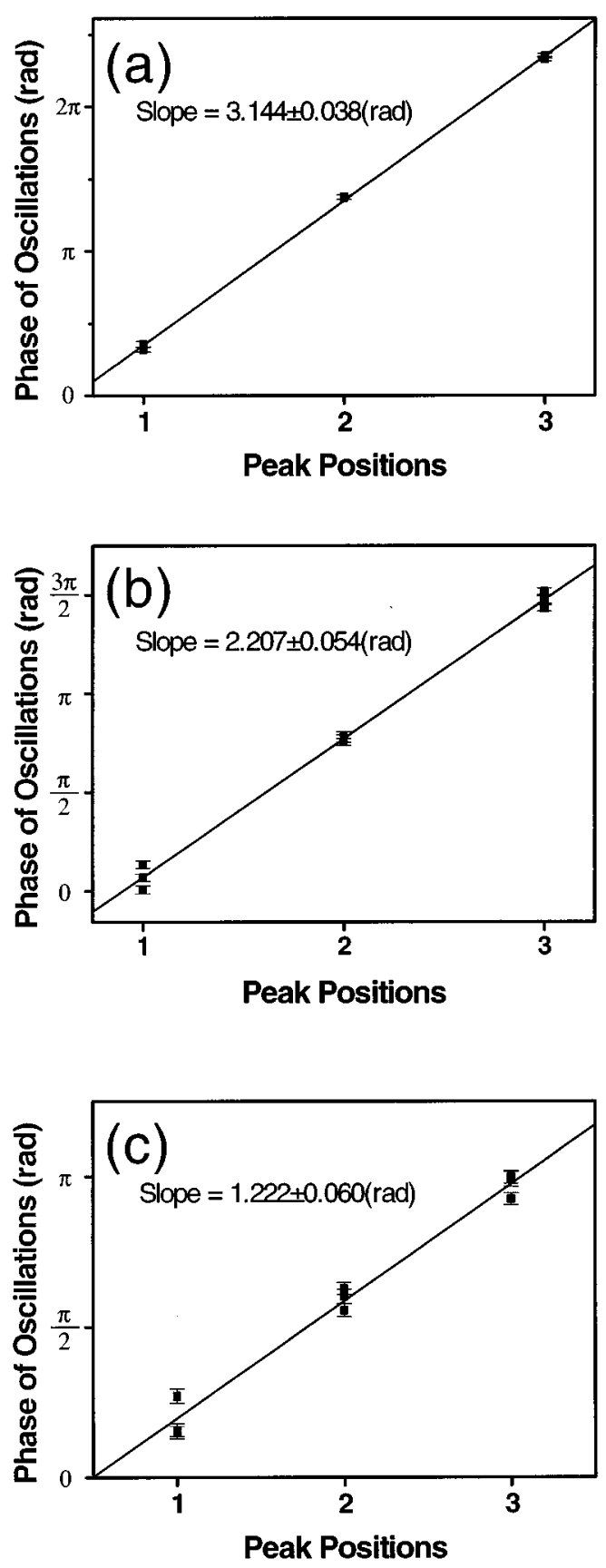

FIG. 6. Fitted shifts of the oscillations for the three measurements (a)-(c) in Fig. 5. The slopes of these plots represent the phase shifts of the out-going beam from the interference loop $A$ for a one-cycle evolution.

\section{Results with the phase shifter II}

The geometric phase shifts, which we intended to measure, were induced on the recombined beam from the interference loop $A$ with the phase shifter I and the absorber. These geometric phase shifts were measured with the phase shifter II in the reference beam. Here, since we pay particular attention to the phase shift by the cyclic evolution of the system, interference oscillations caused by the PS-II were collected by fixing the PS-I at the three peak positions of the intensity modulations as shown in Fig. 4, i.e., at peak 1, peak 2 , and peak 3 . The collected data were fitted to sinusoidal

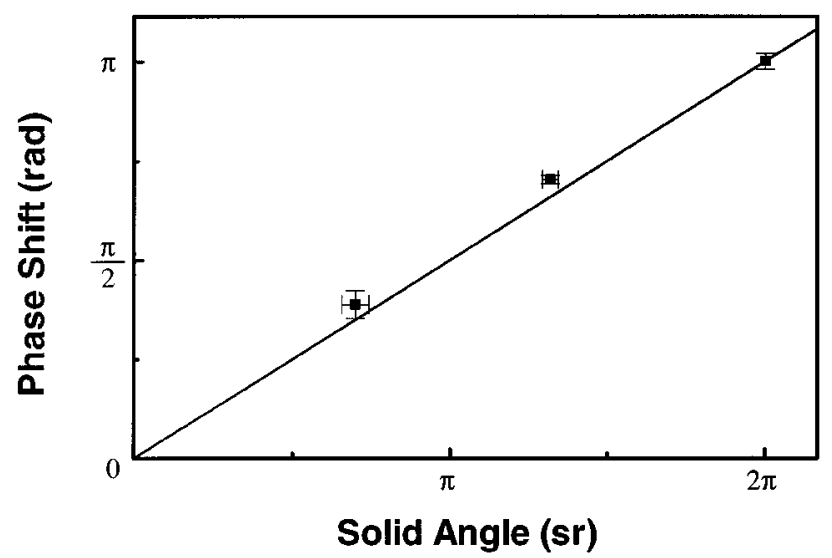

FIG. 7. Experimental results of geometric phase shift as a function of solid angle in the split-beam experiment. The solid line corresponds to the theoretical prediction given by Eq. (9) for $\sigma=$ $\frac{1}{2}$.

curves by the least-squares method. The typical contrast of the oscillations was about $20 \%$ above the background. The intensity oscillations obtained by the PS-II, along with fitting curves and their shifts in peak position, are shown in Fig. 5 for the three different pairs of phase shifters I and absorbers. One can see that the oscillations get shifted depending on the peak positions by the PS-I. In addition, Fig. 5 clearly shows that the magnitude of the shifts in the interference oscillations is reduced commensurate with the decrease in the transmission probability of the absorber.

We collected the data by repeating the same measurements twice for the case (a) of the 10-mm-thick PS-I without an absorber and three times for the cases (b) of the $10 \mathrm{~mm} / 5$ $\mathrm{mm}$, as well as (c) of the $10 \mathrm{~mm} / 2 \mathrm{~mm}$ thick PS-I, each with their respective absorbers. The obtained intensity modulations were fitted to sinusoidal curves and the shifts of the oscillations were analyzed quantitatively. In Fig. 6, the phases of the fitted sinusoidal oscillations are plotted against the peak positions, i.e., peak 1 , peak 2 , and peak 3 , together with the fitted straight lines. The slopes of these lines were obtained by a linear regression fitting procedure. From these slopes, we obtained the shifts of 3.144(38), 2.207(54), and $1.222(60) \mathrm{rad}$ for the cases of the 10-mm-thick PS-I without an absorber, the $10 \mathrm{~mm} / 5 \mathrm{~mm}$ thick PS-I with the absorber $(T=0.49)$ and the $10 \mathrm{~mm} / 2 \mathrm{~mm}$ thick PS-I with the absorber $(T=0.21)$, respectively.

In the theoretical predictions, the obtained phase shift is associated with the solid angle subtended by the closed curve of the cyclic evolution on the sphere, when this phase shift has a true geometric property. The solid angles $\Omega$ for three combinations of phase shifters I and absorbers are given by Eq. (8). Figure 7 shows the extent of the quantitative agreement between the measured values (squares) and the expected values of the geometric phase shift (solid line) for $\sigma=$ $\frac{1}{2}$ in Eq. (9). A slight deviation from the theory may be due to the fact that the dynamical phase factor was not exactly zero under our experimental conditions.

\section{CONCLUDING REMARKS}

We have derived the geometric phase for a split-beam experiment, and all results obtained thus far are in complete 
agreement with the theoretical predictions. It is clear that the geometric phase for the split-beam experiment is proportional to the solid angle subtended by the curve at the origin and that its coefficient is one-half. This is due to the fact that the spin one half system is closely related to SU(2) [29] and that the cycle of the transformation in the split-beam experiment is also a sequence of $\mathrm{SU}(2)$ transformations $[23,30]$, i.e., rotations. Since the geometric phase in the split-beam experiment is independent of the spin of the beam, the same results could be obtained not only with neutrons but with any kind of particle beam, such as photons, $\mathrm{x}$ rays, atoms, etc. For instance, the geometric phase in a split-beam experiment for photons (spin 1) would also be given by half the solid angle subtended by the curve of the evolution [13], just as for the spin- $\frac{1}{2}$ particles used here.

In an example of the spinor rotation in a homogeneous magnetic field [9], the Hamiltonian in the rest frame provides a positive energy, $\Delta E(=+\mu B>0)$, for a spin-down state, which induces a negative phase shift, $-\Delta E t / \hbar(<0)$. This phase shift, however, can be regarded as positive due to the $2 \pi$ periodicity of the phase. In other words, while the positive energy due to the Hamiltonian causes a negative phase shift in naive considerations, it can be considered to cause a positive phase shift as well. Thus, positive and a negative energies as well as phase shifts, they being intuitively regarded to cancel each other, result in an additional phase factor, namely the geometric phase factor, in the recombined beam. In our experiments, the PS-II induced the phase shifts of about $\pi, \frac{2}{3} \pi$, and $\frac{1}{3} \pi$ to one of the beams, and of about $-\pi,-\frac{4}{3} \pi$, and $-\frac{5}{3} \pi$ to the other for one cycle of the evolu- tion. Although they cancel each other according to Eq. (5), the latter negative phase shifts are equivalent to those of $\pi$, $\frac{2}{3} \pi$, and $\frac{1}{3} \pi$ and the recombined beam gets the geometric phase shifts of about $\pi, \frac{2}{3} \pi$, and $\frac{1}{3} \pi$.

A conscientious reader may point out that these experimental results could be entirely explained using the Schrödinger equation only. One would obtain the same theoretical predictions for the experimental results without the term for the geometric phase. This seems to be a general feature of this kind of phenomenon and is correct in the case of the split-beam experiment, too. The geometrical phase formalism is useful because it sometimes plays a role in fundamental quantum-mechanical phenomena, e.g., Aharanov-Bohm (AB) phase $[31,9]$ or in the noncommutation of Pauli spin operators $[32,33]$.

To the best of our knowledge, this is the first application of the four-plate neutron interferometer for a fundamental measurement. This type of interferometer can be used either for photons or neutrons and is well suited for other fundamental physics applications such as observations of multiple beam mixing phenomena and enhanced squeezing phenomena.

\section{ACKNOWLEDGMENTS}

The hospitality of the Hahn-Meitner Institut is gratefully acknowledged. We thank H. Kaiser and J. Summhammer for critical reading of the manuscript. This work was supported by "Fonds zur Förderung der Wissenschaftlichen Forschung" in Austria (Project No. P8456).
[1] M. V. Berry, Proc. R. Soc. London Ser. A 392, 45 (1984).

[2] B. Simon, Phys. Rev. Lett. 51, 2176 (1983).

[3] S. Pancharatnam, Proc. Indian Acad. Sci. A44, 247 (1956); reprinted in Collected Works of S. Pacharatnam, edited by G. W. Series (Oxford University Press, London, 1975).

[4] A. Tomita and R. Y. Chiao, Phys. Rev. Lett. 57, 937 (1986).

[5] T. Bitter and D. Dubbers, Phys. Rev. Lett. 59, 251 (1987).

[6] D. J. Richardson, A. I. Kilvington, K. Green, and S. K. Lamoreaux, Phys. Rev. Lett. 61, 2030 (1988).

[7] R. Tycko, Phys. Rev. Lett. 58, 2281 (1987).

[8] D. M. Bird and A. R. Preston, Phys. Rev. Lett. 61, 2863 (1988).

[9] Y. Aharanov and J. Anandan, Phys. Rev. Lett. 58, 1593 (1987).

[10] R. Bhandari and J. Samuel, Phys. Rev. Lett. 60, 1211 (1988).

[11] R. Y. Chiao, A. Antaramian, K. M. Ganga, H. Jiao, S. R. Wilkinson, and H. Nathel, Phys. Rev. Lett. 60, 1214 (1988).

[12] D. Suter, K. T. Muller, and A. Pines, Phys. Rev. Lett. 60, 1218 (1988)

[13] R. Simon, H. J. Kimbe, and E. C. G. Sudarshan, Phys. Rev. Lett. 61, 19 (1988).

[14] H. Weinfurter and G. Badurek, Phys. Rev. Lett. 64, 1318 (1990).

[15] M. Reich, U. Sterr, and W. Ertmer, Phys. Rev. A 47, 2518 (1993).

[16] H.-M. Lauber, P. Weidenhammer, and D. Dubbers, Phys. Rev. Lett. 72, 1004 (1994).
[17] Neutron Interferometry, edited by U. Bonse and H. Rauch (Clarendon, Oxford, 1979).

[18] A. G. Klein and S. A. Werner, Rep. Prog. Phys. 46, 259 (1983).

[19] G. Badurek, H. Rauch, and A. Zeilinger (Eds.), "Matter Wave Interferometry" Physica B 151 (1988).

[20] M. Heinrich, D. Petrascheck, and H. Rauch, Z. Phys. B 72, 357 (1988).

[21] A similar experimental situation is described in E. P. Wigner, Am. J. Phys. 31, 6 (1963).

[22] P. Busch, Phys. Rev. D 33, 2253 (1986).

[23] P. Mittelstaedt, A. Pieur, and R. Schieder, Found. Phys. 17, 891 (1987).

[24] P. Busch, Found. Phys. 17, 905 (1987).

[25] Y. Hasegawa and S. Kikuta, Z. Phys. B 93, 133 (1994).

[26] G. Drabkin, A. Ioffe, S. Kursauov, F. Mezei, and V. Zaliyakin, Nucl. Instrum. Methods, Phys. Res. Sect. A 348, 198 (1994).

[27] H. Rauch and J. Summhammer, Phys. Lett. A 104, 44 (1984).

[28] J. Summhammer, H. Rauch, and D. Tuppinger, Phys. Rev. A 36, 4447 (1987).

[29] See, e.g., L. I. Schiff, in Quantum Mechanics, 3rd ed. (McGraw-Hill, New York, 1968), p. 206.

[30] B. Yurke, S. L. McCall, and J. R. Klauder, Phys. Rev. A 33, 4033 (1986).

[31] Y. Aharanov and D. Bohm, Phys. Rev. 115, 485 (1959).

[32] A. G. Wagh, Phys. Lett. A 146, 369 (1990).

[33] Y. Hasegawa (unpublished). 